\title{
Spectral Theory as Influenced by Fritz Gesztesy
}

\author{
Gerald Teschl and Karl Unterkofler \\ To Fritz Gesztesy, teacher, mentor, and friend, on the occasion of his 60th birthday.

\begin{abstract}
We survey a selection of Fritz's principal contributions to the field
\end{abstract} \\ of spectral theory and, in particular, to Schrödinger operators.
}

\section{Introduction}

The purpose of this Festschrift contribution is to highlight some of Fritz's profound contributions to spectral theory and, in particular, to Schrödinger operators. Of course, if you look at his list of publications it is clear that this is a mission impossible and hence the present review will only focus on a small selection. Moreover, this selection is highly subjective and biased by our personal research interests:

- Relativistic Corrections

- Singular Weyl-Titchmarsh-Kodaira Theory

- Inverse Spectral Theory and Trace Formulas

- Commutation Methods

- Oscillation Theory

- Non-self-adjoint operators

Other aspects of his work, e.g., on point interactions and integrable nonlinear wave equations are summarized in the monographs $[2$ and 48,49 , respectively. For some of his seminal contributions to inverse spectral theory [22, 63, 64, 65, 66, 67, 68, we refer to Fritz's own review [38. For some of his recent work on partial differential operators, Krein-type resolvent formulas, operatorvalued Weyl-Titchmarsh operators (i.e., energy-dependent analogs of Dirichlet-toNeumann maps), and Weyl-type spectral asymptotics for Krein-von Neumann extensions of the Laplacian on bounded domains, we refer to $\mathbf{5}, \mathbf{5 4}, \mathbf{5 6}, \mathbf{5 7}, \mathbf{5 8}$, and the detailed lists of references therein.

2010 Mathematics Subject Classification. Primary 35P05, 34L40; Secondary 34B20, 34B24. Key words and phrases. Schrödinger operators, Spectral theory, Oscillation theory.

in "Spectral Analysis, Differential Equations and Mathematical Physics", H. Holden et al. (eds), 343-364, Proceedings of Symposia in Pure Mathematics 87, Amer. Math. Soc., Providence, 2013.

Research supported by the Austrian Science Fund (FWF) under Grant No. Y330 and P24736B23.

(C)2012 (The authors) 
To be sure, this is not a survey of the state of the art on these topics but we rather focus exclusively on Fritz Gesztesy's contributions to and influence exerted on the field. Especially, the bibliography is far from being complete and only reflects the particular purpose of this survey.

Personal note. It was 28 years ago in spring of 1985 that Fritz and Karl first met in Graz. Fritz, jointly with Wolfgang Bulla, advised his master and PhD thesis. Karl then spent two years (1990-1992) as a postdoc with Fritz at the University of Missouri, Columbia. When he returned to Austria, Karl met Gerald, who had just finished his masters with Wolfgang Bulla. Karl immediately proposed to him to pursue his PhD with Fritz. After a brief meeting with Fritz in Graz and a short visit to the University of Missouri, Columbia, Gerald enrolled in the PhD program there and a second successful cooperation began. Fritz guided us through the early stages of our careers and was much more than a mentor for us. On the one hand he was an unlimited source of ideas for new projects and on the other hand he was available for discussing mathematics close to 24 hours at 358 days a year (one week has to be subtracted for which he disappears when snorkeling in the Caribbean or Hawaii). His devotion to mathematics, his perfection when it comes to details, and his endurance has always been a role model for us. And last but not least, he is the one everybody asks for references; his collection of papers and books is legendary!

Happy Birthday, Fritz, and on to many more such anniversaries!

\section{Relativistic Corrections}

In 1950 Foldy and Wouthuysen developed a formal perturbation scheme (which is now "regarded as obsolete", according to Kutzelnigg on page 678 in 121]) to obtain relativistic corrections of the nonrelativistic Pauli operator $H_{+}$. However, adding the first correction term of order $c^{-2}$ already destroys all spectral properties of $H_{+}$. The fact, that nevertheless formal perturbation theory yields correct results in special cases has been explained in terms of spectral concentration in 43 , 39 and $\mathbf{1 3 8}$.

Historically, the first rigorous treatment of the nonrelativistic limit of Dirac Hamiltonians goes back to Titchmarsh [137. who proved holomorphy of the Dirac eigenvalues (rest energy subtracted) with respect to $c^{-2}$ for spherically symmetric potentials and obtained explicit formulas for relativistic bound state corrections of order $\mathrm{O}\left(c^{-2}\right)$ (formally derived in [123.). Holomorphy of the Dirac resolvent in three dimensions in $c^{-1}$ for electrostatic interactions were first obtained by Veselic 139 and then extended to electromagnetic interactions by Hunziker [85. An entirely different approach, based on an abstract set up, has been used in 15 to prove strong convergence of the unitary groups as $c^{-1} \rightarrow 0$. Convergence of solutions of the Dirac equation based on semigroup methods has also been obtained in 120. Time-dependent electromagnetic fields are treated in [7].

In joint collaboration with Bernd Thaller and Harald Grosse [41] (see also 40. and 42 ) employing an abstract framework, holomorphy of the Dirac resolvent in $c^{-1}$ under very general conditions on the electromagnetic interaction potentials was obtained. Moreover, this approach led to the first rigorous derivation of explicit formulas for relativistic corrections of order $\mathrm{O}\left(c^{-2}\right)$ to bound state energies.

Application of these results lead to relativistic corrections for energy bands and corresponding corrections for impurity bound states for one-dimensional periodic 
systems 11 and relativistic corrections for the scattering matrix [12. An explicit treatment of relativistic corrections of the scattering amplitude appeared in $\mathbf{8 3}$.

A thorough and comprehensive treatment of Dirac operators can be found in the book by Thaller [136] and in 121.

Based on the abstract approach introduced in $\mathbf{8 5}$ and $\mathbf{1 5}$, let $\mathcal{H}_{ \pm}$be separable, complex Hilbert spaces. One introduces self-adjoint operators $\alpha, \beta$ in $\mathcal{H}=$ $\mathcal{H}_{+} \oplus \mathcal{H}_{-}$of the type

$$
\alpha=\left(\begin{array}{cc}
0 & A^{*} \\
A & 0
\end{array}\right), \quad \beta=\left(\begin{array}{cc}
1 & 0 \\
0 & -1
\end{array}\right),
$$

where $A$ is a densely defined, closed operator from $\mathcal{H}_{+}$into $\mathcal{H}_{-}$. Next, define the abstract free Dirac operator $H^{0}(c)$ by

$$
H^{0}(c)=c \alpha+m c^{2} \beta, \quad \operatorname{dom}\left(H^{0}(c)\right)=\operatorname{dom}(\alpha), \quad c \in \mathbb{R} \backslash\{0\}, \quad m>0
$$

and the interaction $V$ by

$$
V=\left(\begin{array}{cc}
V_{+} & 0 \\
0 & V_{-}
\end{array}\right)
$$

where $V_{ \pm}$denote self-adjoint operators in $\mathcal{H}_{ \pm}$, respectively. Assuming $V_{+}$(resp. $V_{-}$) to be bounded w.r.t. $A$ (resp. $\left.A^{*}\right)$, i.e.,

$$
\operatorname{dom}(A) \subseteq \operatorname{dom}\left(V_{+}\right), \quad \operatorname{dom}\left(A^{*}\right) \subseteq \operatorname{dom}\left(V_{-}\right),
$$

the abstract Dirac operator $H(c)$ reads

$$
H(c)=H^{0}(c)+V, \quad \operatorname{dom}(H(c))=\operatorname{dom}(\alpha) .
$$

Obviously, $H(c)$ is self-adjoint for $|c|$ large enough. The corresponding self-adjoint (free) Pauli operators in $\mathcal{H}_{ \pm}$are then defined by

$$
\begin{array}{lll}
H_{+}^{0}=(2 m)^{-1} A^{*} A, & H_{+}=H_{+}^{0}+V_{+}, & \operatorname{dom}\left(H_{+}\right)=\operatorname{dom}\left(A^{*} A\right), \\
H_{-}^{0}=(2 m)^{-1} A A^{*}, & H_{-}=H_{-}^{0}+V_{-}, & \operatorname{dom}\left(H_{-}\right)=\operatorname{dom}\left(A A^{*}\right) .
\end{array}
$$

Introducing the operator $B(c)$ (see 85$]$ )

$$
B(c)=\left(\begin{array}{ll}
1 & 0 \\
0 & c
\end{array}\right),
$$

one establishes the holomorphy of the Dirac resolvent in $c^{-1}$.

TheOREM 2.1. Let $H(c)$ be defined as above and fix $z \in \mathbb{C} \backslash \mathbb{R}$. Then (a) $\left(H(c)-m c^{2}-z\right)^{-1}$ is holomorphic w.r.t. $c^{-1}$ around $c^{-1}=0$

$$
\begin{aligned}
& \left(H(c)-m c^{2}-z\right)^{-1} \\
& =\left\{1+\left(\begin{array}{cc}
0 & (2 m c)^{-1}\left(H_{+}-z\right)^{-1} A^{*}\left(V_{-}-z\right) \\
(2 m c)^{-1} A\left(H_{+}^{0}-z\right)^{-1} V_{+} & \left(2 m c^{2}\right)^{-1} z\left(H_{-}^{0}-z\right)^{-1}\left(V_{-}-z\right)
\end{array}\right)\right\}^{-1} \times \\
& \times\left(\begin{array}{cc}
\left(H_{+}-z\right)^{-1} & (2 m c)^{-1}\left(H_{+}-z\right)^{-1} A^{*} \\
(2 m c)^{-1} A\left(H_{+}^{0}-z\right)^{-1} & \left(2 m c^{2}\right)^{-1} z\left(H_{-}^{0}-z\right)^{-1}
\end{array}\right) .
\end{aligned}
$$


(b) $B(c)\left(H(c)-m c^{2}-z\right)^{-1} B(c)^{-1}$ is holomorphic w.r.t. $c^{-2}$ around $c^{-2}=0$

$$
\begin{aligned}
& B(c)\left(H(c)-m c^{2}-z\right)^{-1} B(c)^{-1} \\
& =\left\{1+\left(\begin{array}{cc}
0 & \left(2 m c^{2}\right)^{-1}\left(H_{+}-z\right)^{-1} A^{*}\left(V_{-}-z\right) \\
0 & \left(2 m c^{2}\right)^{-1}\left[(2 m)^{-1} A\left(H_{+}-z\right)^{-1} A^{*}-1\right]\left(V_{-}-z\right)
\end{array}\right)\right\}^{-1} \times \\
& \times\left(\begin{array}{cc}
\left(H_{+}-z\right)^{-1} & \left(2 m c^{2}\right)^{-1}\left(H_{+}-z\right)^{-1} A^{*} \\
(2 m)^{-1} A\left(H_{+}-z\right)^{-1} & \left(2 m c^{2}\right)^{-1}\left[(2 m)^{-1} A\left(H_{+}-z\right)^{-1} A^{*}-1\right]
\end{array}\right) .
\end{aligned}
$$

First order expansions in (2.8) and (2.9) yield

$$
\begin{aligned}
& \left(H(c)-m c^{2}-z\right)^{-1}=\left(\begin{array}{cc}
\left(H_{+}-z\right)^{-1} & 0 \\
0 & 0
\end{array}\right) \\
& +c^{-1}\left(\begin{array}{cc}
0 & (2 m)^{-1}\left(H_{+}-z\right)^{-1} A^{*} \\
(2 m)^{-1} A\left(H_{+}-z\right)^{-1} & 0
\end{array}\right)+O\left(c^{-2}\right)
\end{aligned}
$$

(clearly illustrating the nonrelativistic limit $|c| \rightarrow \infty$ ) and

$$
\begin{aligned}
& B(c)\left(H(c)-m c^{2}-z\right)^{-1} B(c)^{-1}=: R_{0}(z)+c^{-2} R_{1}(z)+O\left(c^{-4}\right) \\
& =\left(\begin{array}{cr}
\left(H_{+}-z\right)^{-1} & 0 \\
(2 m)^{-1} A\left(H_{+}-z\right)^{-1} & 0
\end{array}\right)+c^{-2}\left(\begin{array}{ll}
R_{11}(z) & R_{12}(z) \\
R_{21}(z) & R_{22}(z)
\end{array}\right)+O\left(c^{-4}\right),
\end{aligned}
$$

where

$$
\begin{aligned}
& R_{11}(z)=(2 m)^{-2}\left(H_{+}-z\right)^{-1} A^{*}\left(z-V_{-}\right) A\left(H_{+}-z\right)^{-1}, \\
& R_{12}(z)=(2 m)^{-1}\left(H_{+}-z\right)^{-1} A^{*}, \\
& R_{21}(z)=(2 m)^{-2}\left((2 m)^{-1} A\left(H_{+}-z\right)^{-1} A^{*}-1\right)\left(z-V_{-}\right) A\left(H_{+}-z\right)^{-1}, \\
& R_{22}(z)=(2 m)^{-1}\left((2 m)^{-1} A\left(H_{+}-z\right)^{-1} A^{*}-1\right) .
\end{aligned}
$$

To formulate and prove Theorem 2.1 Fritz et al. combined a clever decomposition of the Dirac resolvent with a skillful application of some commutations formulas by Deift [20] (see also Section 5).

Analyzing the relationship between the spectrum of $\left(H_{+}-z\right)^{-1}$ and $R_{0}(z)$ now yields the following result on relativistic eigenvalue corrections.

TheOrem 2.2. Let $H(c)$ be defined as in (2.5) and assume $E_{0} \in \sigma_{d}\left(H_{+}\right)$to be a discrete eigenvalue of $H_{+}$of multiplicity $m_{0} \in \mathbb{N}$. Then, for $c^{-2}$ small enough, $H(c)-m c^{2}$ has precisely $m_{0}$ eigenvalues (counting multiplicity) near $E_{0}$ which are all holomorphic w.r.t. $c^{-2}$. More precisely, all eigenvalues $E_{j}\left(c^{-2}\right)$ of $H(c)-m c^{2}$ near $E_{0}$ satisfy

$$
E_{j}\left(c^{-2}\right)=E_{0}+\sum_{k=1}^{\infty}\left(c^{-2}\right)^{k} E_{j, k}, \quad j=1, \ldots, j_{0}, \quad j_{0} \leq m_{0}
$$

and if $m_{j}$ denotes the multiplicity of $E_{j}\left(c^{-2}\right)$ then $\sum_{j=1}^{j_{0}} m_{j}=m_{0}$.

In addition, there exist linearly independent vectors

$$
f_{j l}\left(c^{-1}\right)=\left(\begin{array}{c}
f_{+j l}\left(c^{-2}\right) \\
c^{-1} f_{-j l}\left(c^{-2}\right)
\end{array}\right), \quad j=1, \ldots j_{0}, \quad l=1, \ldots, m_{j}
$$

s.t. $f_{ \pm j i}$ are holomorphic w.r.t. $c^{-2}$ near $c^{-2}=0$ and

$$
H_{+} f_{+j l}(0)=E_{0} f_{+j l}(0), \quad f_{-j l}(0)=(2 m)^{-1} A f_{+j l}(0)
$$


and

$$
\left(H(c)-m c^{2}\right) f_{j l}\left(c^{-1}\right)=E_{j}\left(c^{-2}\right) f_{j l}\left(c^{-1}\right), \quad j=1, \ldots, j_{0}, \quad l=1, \ldots, m_{j} .
$$

The eigenvectors $f_{j l}\left(c^{-1}\right)$ can be chosen to be orthonormal. Finally, the first-order corrections $E_{j, 1}$ in (2.12) are explicitly given as the eigenvalues of the matrix

$$
(2 m)^{-2}\left(A f_{r},\left(V_{-}-E_{0}\right) A f_{s}\right), \quad r, s=1, \ldots \ldots, m_{0},
$$

where $\left\{f_{r}\right\}_{r=1}^{m_{0}}$ is any orthonormal basis of the eigenspace of $H_{+}$to the eigenvalue $E_{0}$.

Remarks: (a) The main idea behind Theorem 2.2 was to look for eigenvalues of the resolvent $\left(H(c)-m c^{2}-z\right)^{-1}$ and applying the strong spectral mapping theorem ([109], page 109, Lemma 2) instead of looking directly for eigenvalues of the unbounded Hamiltonian $H(c)-m c^{2}$.

(b) Theorem 2.2 for $m_{0}=1$ is due to Fritz et al. (see [41, 40]). In the general case $m_{0}>1$ only holomorphy of $E_{j}\left(c^{-1}\right)$ w.r.t. $c^{-1}$ near $c^{-1}=0$ and $E_{j}\left(c^{-1}\right)-m c^{2}-E_{0}={ }_{c \rightarrow \infty} O\left(c^{-2}\right)$ has been proven in 41]. The above extension of this result for $m_{0}>1$ is due to $\mathbf{1 4 4}$.

The basic idea to prove holomorphy of $E_{j}\left(c^{-1}\right)$ w.r.t. $c^{-2}$ is the following: Since $\left(H(c)-m c^{2}-z\right)^{-1}$ is normal for $z \in \mathbb{C} \backslash \mathbb{R},(2.10)$ implies that the projection $P_{j}\left(c^{-1}\right)$ onto the eigenspace of the eigenvalue $\left(E_{j}\left(c^{-1}\right)-z\right)^{-1}$ is holomorphic w.r.t. $c^{-1}$ near $c^{-1}=0$. To prove that $E_{j}\left(c^{-1}\right)$ is actually holomorphic w.r.t. $c^{-2}$ near $c^{-2}=0$ one calculates

$$
\begin{aligned}
& \tilde{P}_{j}\left(c^{-2}\right):=B(c) P_{j}\left(c^{-1}\right) B(c)^{-1}=\left(\begin{array}{cc}
1 & 0 \\
0 & c
\end{array}\right)\left(\begin{array}{cc}
p_{j} & 0 \\
0 & 0
\end{array}\right)\left(\begin{array}{cc}
1 & 0 \\
0 & 1 / c
\end{array}\right) \\
& +\left[\text { terms holomorphic w.r.t. } c^{-2}\right]=\left[\text { terms holomorphic w.r.t. } c^{-2}\right] .
\end{aligned}
$$

Here $\tilde{P}_{j}\left(c^{-2}\right)$ and $p_{j}$ are the corresponding projections associated with $B(c)\left(H(c)-m c^{2}-z\right)^{-1} B(c)^{-1}$ and $\left(H_{+}-z\right)^{-1}$ of dimension $m_{j}$, respectively. Thus, $\left\|\tilde{P}_{j}\left(c^{-2}\right)\right\|$ is bounded as $c^{-2} \rightarrow 0$ and, hence, Butler's theorem ([90], p. 70) proves that $\tilde{P}_{j}\left(c^{-2}\right)$ and $\left(E_{j}\left(c^{-1}\right)-z\right)^{-1}$ are actually holomorphic w.r.t. $c^{-2}$ near $c^{-2}=0$.

\section{Singular Weyl-Titchmarsh-Kodaira Theory}

The purpose of the remaining sections is to survey some highlights obtained by Fritz for one-dimensional Schrödinger operators

$$
H=-\frac{d^{2}}{d x^{2}}+q, \quad \text { in } \mathcal{H}=L^{2}(a, b), \quad-\infty \leq a<b \leq \infty .
$$

In what follows we will denote by $\tau$ the underlying differential operator and by $H$ an associated self-adjoint operator (as determined by separated boundary conditions whenever necessary, i.e., when $\tau$ is in the limit circle case at one of the endpoints). We will assume that the reader is familiar with the basic results from spectral theory for these operators and refer, e.g., to the textbooks [102, 134, 140, 142.

One key object in direct and inverse spectral theory is the Weyl-Titchmarsh $m$-function. To define it suppose that the endpoint $a$ is regular, that is $a$ is finite and all solutions (as well as their derivatives) extend continuously to this endpoint. For simplicity take a Dirichlet boundary condition, $f(a)=0$, at $a$. Then we can choose a basis of solutions of $\tau u=z u$ satisfying the initial conditions

$$
\theta(z, a)=\phi^{\prime}(z, a)=1, \quad \theta^{\prime}(z, a)=\phi(z, a)=0 .
$$


In particular, $\phi(z, x)$ satisfies the Dirichlet boundary condition at $a$. Moreover, for $z \in \mathbb{C} \backslash \mathbb{R}$ there will also be a unique (up to scaling) solution $\psi(z, x)$ which will be square integrable near the other end point $b$ and which satisfies a possible boundary condition at $b$. If we normalize this function according to $\psi(z, a)=1$ it can be written as the following linear combination of our basis of solutions

$$
\psi(z, x)=\theta(z, x)+m(z) \phi(z, x),
$$

where the Weyl-Titchmarsh $m$-functions is given by

$$
m(z)=\frac{\psi^{\prime}(z, a)}{\psi(z, a)} .
$$

It is a key result that $m(z)$ is a Herglotz-Nevanlinna function (i.e., an analytic function mapping the upper half plane into itself) and hence it can be represented as

$$
m(z)=\operatorname{Re}(m(\mathrm{i}))+\int_{\mathbb{R}}\left(\frac{1}{\lambda-z}-\frac{\lambda}{1+\lambda^{2}}\right) d \rho(\lambda),
$$

where $\rho$ is a Borel measure with $\int_{\mathbb{R}}\left(1+\lambda^{2}\right)^{-1} d \rho(\lambda)<\infty$ given by the Stieltjes inversion formula

$$
\frac{1}{2}\left(\rho\left(\left(\lambda_{0}, \lambda_{1}\right)\right)+\rho\left(\left[\lambda_{0}, \lambda_{1}\right]\right)\right)=\lim _{\varepsilon \downarrow 0} \frac{1}{\pi} \int_{\lambda_{0}}^{\lambda_{1}} \operatorname{Im}(m(\lambda+\mathrm{i} \varepsilon)) d \lambda .
$$

Moreover, the map

$$
f(x) \mapsto \hat{f}(\lambda)=\int_{a}^{b} \phi(\lambda, x) f(x) d x,
$$

initially defined on functions $f$ with compact support in $[a, b)$, extends to a unitary map from $L^{2}(a, b)$ to $L^{2}(\mathbb{R}, d \rho)$ which maps our operator $H$ to multiplication with the independent variable $\lambda$. In particular, the spectral measure $\rho$ contains all the spectral information of $H$ ! In his paper with Zinchenko [82, Fritz not only gave a particularly simple proof of this fact but also pointed out a crucial extension which is now known as singular Weyl-Titchmarsh-Kodaira theory.

In fact, the assumption that one endpoint is regular excludes many important examples which have a simple spectrum and where such a transformation should still exist by the spectral theorem. Two examples of central interest in quantum mechanics are the radial part of the Coulomb Hamiltonian, where $q(x)=\frac{l(l+1)}{x^{2}}-$ $\frac{\gamma}{x}$ on $(0, \infty)$, or the harmonic oscillator, $q(x)=x^{2}$ on $(-\infty, \infty)$. In both cases it is still possible to define an entire (w.r.t. $z$ ) solution $\phi(z, x)$ which is square integrable near the left endpoint $a$ and satisfies a possible boundary condition there. Moreover, a singular Weyl $m$-function can be defined as before once a second linearly independent solution $\theta(z, x)$ is chosen. In fact, this observation was already made by Kodaira 91 . However, as pointed out by Kac $\mathbf{8 8}$, this is nontrivial if $\theta(z, x)$ is required to be entire as well. In fact, we can always choose a second entire solution but the Wronskian $W(\theta(z), \phi(z))=\theta(z, x) \phi^{\prime}(z, x)-\theta^{\prime}(z, x) \phi(z, x)$ might have zeros! In 82 this problem was overcome by showing that to define the spectral measure via (3.6) it suffices if $\theta(z, x)$ is analytic in a neighborhood of the real line. And it is easy to check that if $\theta_{c}(z, x), \phi_{c}(z, x)$ are two entire solutions defined by the initial conditions $\theta_{c}(z, c)=\phi_{c}^{\prime}(z, c)=1$ and $\theta_{c}^{\prime}(z, c)=\phi_{c}(z, c)=0$, then

$$
\theta(z, x)=\frac{\phi^{\prime}(z, c)}{\phi(z, c)^{2}+\phi^{\prime}(z, c)^{2}} \theta_{c}(z, x)+\frac{\phi(z, c)}{\phi(z, c)^{2}+\phi^{\prime}(z, c)^{2}} \phi_{c}(z, x)
$$


will do the trick. In fact, by construction it is meromorphic in $\mathbb{C}$ with all poles away from the real axis and one easily checks $W(\theta(z), \phi(z))=1$ (evaluate the Wronskian at $x=c$ and recall that it is independent of $x$ ).

Theorem $3.1(\mathbf{8 2})$. Suppose $H$ has an entire solution $\phi(z, x)$ which is square integrable near the left endpoint a and satisfies a possible boundary condition there. Let $\theta(z, x)$ be defined as in (3.8).

Then there exists a Weyl m-function which is meromorphic in $\mathbb{C} \backslash \mathbb{R}$ (without any poles accumulating near the real line) defined via (3.3) and an associated spectral measure defined via (3.6). Moreover, the map (3.7) initially defined on functions $f$ with compact support in $[a, b)$, extends to a unitary map from $L^{2}(a, b)$ to $L^{2}(\mathbb{R}, d \rho)$ which maps our operator $H$ to multiplication with the independent variable $\lambda$.

The elegant proof from $\mathbf{8 2}$ alluded to before now simply involves the following two well-known facts: First, the resolvent of $H$ which is given by

$$
(H-z)^{-1} f(x)=\int_{a}^{b} G(z, x, y) f(y) d y,
$$

where

$$
G(z, x, y)= \begin{cases}\phi(z, x) \psi(z, y), & y \geq x \\ \phi(z, y) \psi(z, x), & y \leq x\end{cases}
$$

And second, Stone's formula

$$
\left.\langle f, F(H) f\rangle=\frac{1}{\pi} \lim _{\varepsilon \downarrow 0} \int_{\mathbb{R}} F(\lambda) \operatorname{Im}\left\langle f,(H-\lambda-\mathrm{i} \varepsilon)^{-1} f\right\rangle\right) d \lambda
$$

for any bounded continuous function $F \in C_{b}(\mathbb{R})$. Now insert the definitions and compute the limit to obtain

$$
\langle f, F(H) f\rangle=\int_{\mathbb{R}} F(\lambda)|\hat{f}(\lambda)|^{2} d \rho(\lambda),
$$

which in turn implies the theorem.

This seminal result initiated the development of Weyl-Titchmarsh theory at a singular endpoint and has triggered a number of results recently [24, 25, 26, 27, 28, 31, 32, 33, 93, 92, 94, 95, 99.

\section{Inverse Spectral Theory and Trace Formulas}

Next we turn to trace formulas, another area where Fritz has made profound contributions. To this end suppose that $(a, b)=\mathbb{R}$ and that the potential $q(x)$ is periodic: $q(x+1)=q(x)$. Then it is well known by Floquet theory that the spectrum consists of an infinite number of bands

$$
\sigma(H)=\left[E_{0}, E_{1}\right] \cup\left[E_{2}, E_{3}\right] \cup \cdots,
$$

where $E_{0}<E_{1} \leq E_{2}<E_{3} \leq \cdots$. Moreover, if we restrict our operator to the interval $(x, x+1)$ and impose Dirichlet boundary conditions $f(x)=f(x+1)=0$ at the endpoints, then we obtain a sequence of eigenvalues $\mu_{1}(x)<\mu_{2}(x)<\cdots$ depending on the base point $x$. Again it follows from Floquet theory that the Dirichlet eigenvalues $\mu_{j}(x)$ lie in the closures of the spectral gaps: $E_{2 j-1} \leq \mu_{j}(x) \leq$ $E_{2 j}$. 
Then the following trace formula

$$
q(x)=E_{0}+\sum_{j=1}^{\infty}\left[E_{2 j-1}+E_{2 j}-2 \mu_{j}(x)\right]
$$

holds under some suitable assumptions on the potential (e.g., $q \in C^{1}$ ). This trace formula was first obtained in the case where the spectrum has only finitely many gaps (and thus the above sum is finite) and later on generalized to periodic and even some classes of almost-periodic potentials. Furthermore, trace formulas were also known in the context of scattering theory, but in this case the right-hand side contains both a sum corresponding to the eigenvalues plus an integral corresponding to the continuous spectrum. Hence, Fritz was asking for a generalization of (4.2) to more general potentials.

To this end let us briefly sketch one way of proving (4.2). Denote by $\psi_{ \pm}(z, x)$ the Floquet solution of the underlying differential equation and recall that the diagonal Green function is given by

$$
G(z, x, x)=\frac{\psi_{+}(z, x) \psi_{-}(z, x)}{W\left(\psi_{+}(z), \psi_{-}(z)\right)}
$$

where

$$
W_{x}(f, g)=f(x) g^{\prime}(x)-f^{\prime}(x) g(x)
$$

denotes the Wronskian of two absolutely continuous functions. Recall that the Wronskian of two solutions corresponding to the same spectral parameter $z$ is independent of $x$. Then, using the fact that solutions of the underlying differential equation $\tau u=z u$ corresponding to constant initial conditions are entire functions of order one half, one can obtain the following product representation

$$
G(z, x, x)=\frac{1}{2 \sqrt{E_{0}-z}} \prod_{j=1}^{\infty} \frac{z-\mu_{j}(x)}{\sqrt{\left(E_{2 j-1}-z\right)\left(E_{2 j}-z\right)}} .
$$

Comparing this product representation with the well-known asymptotics

$$
G(z, x, x)=\frac{1}{2 \sqrt{-z}}\left(1+\frac{q(x)}{2 z}+o\left(z^{-1}\right)\right)
$$

establishes (4.2).

How can one possibly generalize this argument? Since (4.6) holds for general potentials, a generalization of (4.5) needs to be found and, as observed by Fritz, the correct starting point is the following exponential Herglotz representation

$$
G(z, x, x)=|G(\mathrm{i}, x, x)| \exp \left(\int_{\mathbb{R}}\left(\frac{1}{\lambda-z}-\frac{\lambda}{1+\lambda^{2}}\right) \xi(\lambda, x) d \lambda\right) .
$$

Here the $\xi$-function is given by the Stieltjes inversion formula

$$
\xi(\lambda, x)=\lim _{\varepsilon \downarrow 0} \frac{1}{\pi} \arg G(\lambda+\mathrm{i} \varepsilon, x, x),
$$

where the limit exists for a.e. $\lambda \in \mathbb{R}$. Note that since $G(., x, x)$ is a HerglotzNevanlinna function, we have $0 \leq \xi(\lambda, x) \leq 1$.

Now a trace formula which works for arbitrary potentials follows by comparing asymptotics as before. This is one of the key results obtained together with Barry Simon in 62 . 
TheOREM $4.1(\mathbf{6 2}])$. Suppose $q$ is continuous and bounded from below. Choose $E_{0} \leq \inf \sigma(H)$. Then

$$
V(x)=E_{0}+\lim _{\varepsilon \downarrow 0} \int_{E_{0}}^{\infty} \mathrm{e}^{-\varepsilon \lambda}(1-2 \xi(\lambda, x)) d \lambda .
$$

In fact, even more was shown in 62. Namely, let $H_{x}^{D}=H_{x,-}^{D} \oplus H_{x,+}^{D}$ be the operator $H$ restricted according to $L^{2}(a, b)=L^{2}(a, c) \oplus L^{2}(c, b)$ by imposing Dirichlet boundary conditions at the point $x \in(a, b)$. Then $\xi(\lambda, x)$ was identified as the Krein spectral shift function of the pair $\left(H, H_{x}^{D}\right)$, which in turn opened the door for deriving numerous novel trace formulas for Schrödinger operators: $[37,44,45,46,47,50,51,52,53$.

To demonstrate the usefulness and generality of this theorem let us extract two special cases. First let us see how to obtain (4.2) by evaluating (4.8) using (4.5) (note that the limit is not needed since $G(\lambda, x, x)$ has a continuous extension to the real line away from the band edges). We begin with $\lambda<E_{0}$ in which case $G(\lambda, x, x)$ is positive implying $\xi(\lambda, x)=0$ for $\lambda \in\left(-\infty, E_{0}\right)$. At $E_{0}$ the Green function $G(\lambda, x, x)$ will become purely imaginary implying $\xi(\lambda, x)=\frac{1}{2}$ for $\lambda \in\left(E_{0}, E_{1}\right)$. After $E_{1}$ it will become negative and change sign at its zero $\mu_{1}(x)$, implying $\xi(\lambda, x)=1$ for $\lambda \in\left(E_{1}, \mu_{1}(x)\right)$ and $\xi(\lambda, x)=0$ for $\lambda \in\left(\mu_{1}(x), E_{2}\right)$. Clearly this pattern keeps repeating and we obtain (4.2) (assuming $q \in C^{1}$ the spectral gaps will close sufficiently fast, such that the limit in (4.9) can be taken inside the integral).

A novel application is to confining potentials, like the harmonic oscillator, satisfying $q(x) \rightarrow+\infty$ as $|x| \rightarrow \infty$. Then $H$ has purely discrete spectrum $E_{0}<E_{1}<\cdots$ and the same is true for $H_{x}^{D}$ whose eigenvalues $\mu_{1}(x)<\mu_{2}(x)<\cdots$ are known to satisfy $E_{j-1} \leq \mu_{j}(x) \leq E_{j}$. Then note that $G(z, x, x)$ has first order poles at the eigenvalues $E_{j}$ and first order zeros at the Dirichlet eigenvalues $\mu_{j}(x)$ (since either $\psi_{-}(\lambda, x)$ or $\psi_{+}(\lambda, x)$ must vanish at $\lambda=\mu_{j}(x)$ - if both should vanish they must be linearly dependent and thus such a point must also be an eigenvalue). Hence $G(z, x, x)$ will be real-valued on the real line and change sign at every pole $E_{j}$ and every zero $\mu_{j}(x)$ implying

$$
V(x)=E_{0}+\lim _{\varepsilon \downarrow 0} \frac{1}{\varepsilon} \sum_{j \in \mathbb{N}} 2 \mathrm{e}^{-\varepsilon \mu_{j}(x)}-\mathrm{e}^{-\varepsilon E_{j-1}}-\mathrm{e}^{-\varepsilon E_{j}},
$$

which is just an abelianized version of (4.2).

This brings us to another item discussed in [62, namely the consequences of the above ideas for inverse spectral theory. While the inverse spectral theory is well understood for periodic operators, this is not the case for confining potentials. For example, one open question is to describe the isospectral class of the harmonic oscillator. To see how the $\xi$-function can help understanding this problem, we begin with the observation that $\xi(\lambda, x)$ determines $G(z, x, x)$ by virtue of (4.7) (the unknown constant can be determined from the asymptotics (4.6)).

Now let $\psi_{ \pm}(z, x)$ be the solutions of $\tau u=z u$ which are square integrable near $\pm \infty$ and observe that (3.10) reads

$$
G(z, x, x)=\frac{-1}{m_{+}(z, x)+m_{-}(z, x)},
$$


where

$$
m_{ \pm}(z, x)= \pm \frac{\psi_{ \pm}^{\prime}(z, x)}{\psi_{ \pm}(z, x)}
$$

are the Weyl $m$-functions of $H_{x, \pm}^{D}$. Hence if you fix $x$, say $x=0$, then $\xi(\lambda, 0)$ determines the sum of the spectral measures $\rho_{0, \pm}$ corresponding to $H_{0, \pm}^{D}$ and it remains to split this information into its two pieces (recall that $\rho_{0, \pm}$ determine $H_{0, \pm}^{D}$ and thus $H$ ). Clearly this is impossible in general without additional data. However, it turns out that things get particularly simple if the following reflectionless property is assumed:

$$
m_{-}(\lambda+\mathrm{i} 0, x)=-\overline{m_{-}(\lambda+\mathrm{i} 0, x)}, \quad \text { a.e. } \lambda \in \sigma_{\text {ess }}(H),
$$

for one (it then follows for all) $x$. This covers for example periodic operators, reflectionless potentials from scattering theory (which is where the name comes from), or operators with purely discrete spectrum. In fact, this condition tells us that the absolutely continuous part of the measure needs to be split equally. Moreover, assuming that there is no singularly continuous part it remains to assign a sign $\sigma_{j} \in\{ \pm\}$ to every Dirichlet eigenvalue $\mu_{j}=\mu_{j}(0)$ which encodes if it is an eigenvalue of $H_{0,-}^{D}$ or $H_{0,+}^{D}$ (if it is an eigenvalue of both, which can only happen if it is also an eigenvalue of $H$ as pointed out before, a number $\sigma_{j} \in(-1,1)$ is needed to encode how the mass should be split).

By construction we obtain that the eigenvalues $\left\{E_{j}\right\}_{j \in \mathbb{N}_{0}}$ together with the Dirichlet data $\left\{\left(\mu_{j}, \sigma_{j}\right)\right\}_{j \in \mathbb{N}}$ uniquely determine $H$. The remaining problem to determine the isospectral class of a given operator $H_{0}$ is to describe the set of admissible Dirichlet data. One key contribution towards answering this question is the Dirichlet deformation method to be discusses in the next section, which shows that all restrictions (apart form the obvious ones already listed above) must be of an asymptotic nature since any finite part of the Dirichlet data can be changed at will.

For further information on these circle of ideas see $[\mathbf{1 6}, \mathbf{1 7}, \mathbf{3 7}, 44,45,46,47$, 50, 51, 52, 53, 55, 59, 69, 107, 111, 112, 113, 124, 132, 145. In particular, we mention also the review by Fritz $\mathbf{3 8}$.

\section{Commutation Methods}

Another topic where Fritz made seminal contributions is commutation methods. These are methods of inserting (and removing) eigenvalues in spectral gaps of a given one-dimensional Schrödinger operator $H$. They play a prominent role in diverse fields such as the inverse scattering approach (see, e.g., [21, 103 and the references therein), supersymmetric quantum mechanics (see, e.g., [61 and the references therein), level comparison theorems (cf. 6] and the literature cited therein), and in connection with Bäcklund transformations for the $\mathrm{KdV}$ hierarchy (see, e.g., 73, 61 and the references therein).

Historically, these methods of inserting eigenvalues go back to Jacobi [87. and Darboux [19] with decisive later contributions by Crum [18, Schmincke 118, and, especially, Deift [20]. Two particular methods, shortly to be discussed in an informal manner in (5.1) - (5.5) below, turned out to be of special importance: The single commutation method, also called the Crum-Darboux method [18, [19] (actually going back at least to Jacobi [87]) and the double commutation method, to be found, e.g., in the seminal work of Gel'fand and Levitan [35. 
The single commutation method relies on existence of a positive solution $\psi$ of $H \psi=\lambda \psi$ which confines its applicability to the insertion of eigenvalues below the spectrum of $H$ (assuming $H$ to be bounded from below). Introducing

$$
A=\frac{d}{d x}+\phi, \quad A^{*}=-\frac{d}{d x}+\phi, \quad \text { where } \phi=\frac{d}{d x} \log \psi,
$$

a straightforward calculation reveals

$$
H=A^{*} A+\lambda=-\frac{d^{2}}{d x^{2}}+q, \quad \hat{H}=A A^{*}+\lambda=-\frac{d^{2}}{d x^{2}}+\hat{q},
$$

with

$$
\hat{q}=q-2 \frac{d^{2}}{d x^{2}} \log \psi
$$

Thus (taking proper domain considerations into account) we can define two operators $H, \hat{H}$ on $\mathcal{H}=L^{2}(a, b)$ which turn out unitarily equivalent when restricted to the orthogonal complement of the eigenspaces corresponding to $\lambda$. In fact, using the polar decomposition $A=U|A|$, where $|A|=\left(A^{*} A\right)^{1 / 2}$ and $U: \operatorname{ker}(A)^{\perp} \rightarrow \operatorname{ker}\left(A^{*}\right)^{\perp}$ is unitary, one infers $\hat{H} P=\left(A A^{*}+\lambda\right) P=U|A||A| U^{*}+\lambda P=U H U^{*}$ with $P=U U^{*}$, the projection onto $\operatorname{ker}\left(A^{*}\right)^{\perp}$. Moreover, $H-\lambda, \hat{H}-\lambda \geq 0$ which is equivalent to the existence of the positive solution $\psi$ [81]. Formulas (5.1)-(5.3) constitute the single commutation method.

The double commutation method on the other hand, allows one to insert eigenvalues into any spectral gap of $H$. To this end we assume that $\psi$ is square integrable near $a$ and consider two more expressions $A_{\gamma}, A_{\gamma}^{*}$ as above with $\psi_{\gamma}=$ $\psi /\left(1+\gamma \int_{a}^{x} \psi(t)^{2} d t\right)$. This implies

$$
H=A A^{*}+\lambda=A_{\gamma}^{*} A_{\gamma}+\lambda, \quad H_{\gamma}=A_{\gamma} A_{\gamma}^{*}+\lambda=-\frac{d^{2}}{d x^{2}}+q_{\gamma},
$$

where

$$
q_{\gamma}=q-2 \frac{d^{2}}{d x^{2}} \log \left(1+\gamma \int_{a}^{x} \psi(t)^{2} d t\right) .
$$

The considerations for the single commutation method show that $H$ and $H_{\gamma}$ are unitarily equivalent when restricted to the orthogonal complement of the eigenspaces corresponding to $\lambda$ as long as $\psi$ is positive. However, observe that $q_{\gamma}$ is well defined even if $\psi$ has zeros, and it is natural to conjecture that the last claim continuous to hold even in the case where all intermediate operators are ill-defined. This turned out much harder to prove and was achieved by Fritz [36 on the basis of Weyl-Titchmarsh $m$-function techniques.

In what follows we will denote by $\tau$ the underlying differential operator and by $H$ an associated self-adjoint operator (as determined by separated boundary conditions whenever necessary, i.e., when $\tau$ is in the limit circle case at one of the endpoints).

To this end, suppose $a$ is regular (i.e., $a$ is finite and $q \in L^{1}(a, c)$ such that all solutions extend continuously to the endpoint $a$ ) and introduce the Weyl-Titchmarsh $m$-function as

$$
m(z)=\frac{\psi^{\prime}(z, a)}{\psi(z, a)}
$$

where $\psi(z, x)$ is the unique (up to scaling) solution of $\tau \psi=z \psi$ which is square integrable and satisfies a possible boundary condition at $b$. Then it is well known that $m(z)$ carries all the information on $H$ and hence it suffices to find an explicit 
expression for the Weyl-Titchmarsh $m$-function of $H_{\gamma}$ in terms of the one of $H$. This strategy was carried out in 36 and the following theorem was obtained as one of the main results.

THEOREM $5.1([\mathbf{3 6}, \mathbf{7 1})$. Let $\lambda \in \mathbb{R}$ and $\psi(\lambda,$.$) be a solution satisfying the$ following conditions:

(i) $\psi, \psi^{\prime} \in A C_{l o c}(a, b)$ and $\psi$ is a real-valued solution of $\tau \psi=\lambda \psi$.

(ii) $\psi$ is square integrable near a and fulfills the boundary condition (of $H$ ) at $a$ and $b$ if any (i.e., if $\tau$ is limit circle (l.c.) at a respectively $b$ ).

Define

$$
\psi_{\gamma}(\lambda, x)=\frac{\psi(\lambda, x)}{1+\gamma \int_{a}^{x} \psi(\lambda, t)^{2} d t} .
$$

Let $P(\lambda), P_{\gamma}(\lambda)$ be the projections on the subspaces spanned by $\psi(\lambda), \psi_{\gamma}(\lambda)$, respectively (if one of these functions is not square integrable, set the corresponding projection equal to zero). Then the operator $H_{\gamma}$ defined by

$$
\begin{aligned}
H_{\gamma} f=\tau_{\gamma} f, \quad \operatorname{dom}\left(H_{\gamma}\right)= & \left\{f \in \mathcal{H} \mid f, p f^{\prime} \in A C_{l o c}((a, b)) ; \tau_{\gamma} f \in \mathcal{H} ;\right. \\
& \left.W_{a}\left(\psi_{\gamma}(\lambda), f\right)=W_{b}\left(\psi_{\gamma}(\lambda), f\right)=0\right\},
\end{aligned}
$$

with $q_{\gamma}$ given by (5.5) is self-adjoint. Moreover, $H_{\gamma}$ has the eigenfunction $\psi_{\gamma}(\lambda)$ associated with the eigenvalue $\lambda$. If $\psi(\lambda) \notin \mathcal{H}$ (and hence $\tau$ is limit point (l.p.) at b) we have

$$
H_{\gamma}\left(1-P_{\gamma}(\lambda)\right)=U_{\gamma} H U_{\gamma}^{-1}\left(1-P_{\gamma}(\lambda)\right)
$$

for some unitary operator $U_{\gamma}$ and thus

$$
\begin{aligned}
& \sigma\left(H_{\gamma}\right)=\sigma(H) \cup\{\lambda\}, \quad \sigma_{a c}\left(H_{\gamma}\right)=\sigma_{a c}(H), \\
& \sigma_{p}\left(H_{\gamma}\right)=\sigma_{p}(H) \cup\{\lambda\}, \quad \sigma_{s c}\left(H_{\gamma}\right)=\sigma_{s c}(H) .
\end{aligned}
$$

(Here $\sigma_{a c}(),. \sigma_{s c}($.$) denotes the absolutely and singularly continuous spectrum, re-$ spectively.) If $\psi(\lambda) \in \mathcal{H}$ there is a unitary operator $\tilde{U}_{\gamma}=U_{\gamma} \oplus \sqrt{1+\gamma\|\psi(\lambda)\|^{2}} U_{\gamma}$ on $(1-P(\lambda)) \mathcal{H} \oplus P(\lambda) \mathcal{H}$ such that

$$
H_{\gamma}=\tilde{U}_{\gamma} H \tilde{U}_{\gamma}^{-1}
$$

As already mentioned, the main part of this result is due to Fritz [36. In Gesztesy and Teschl 71 the transformation operator was identified to be

$$
\left(U_{\gamma} f\right)(x)=f(x)-\gamma \psi_{\gamma}(\lambda, x) \int_{a}^{x} \psi(\lambda, t) f(t) d t
$$

and the above theorem reduces to checking that $U_{\gamma}$ has the claimed properties. Moreover, the whole method was extended to Sturm-Liouville operators and some technical assumptions were relaxed. For the connections with singular WeylTitchmarsh-Kodaira theory see $\mathbf{9 3}$.

A further decisive contribution was made by Gesztesy, Simon, and Teschl [70 motivated by a commutation method first introduced by Finkel, Isaacson, and Trubowitz 30 in connection with an explicit realization of the isospectral torus of periodic potentials. This method was again used by Buys and Finkel [13. (see also Iwasaki $[\mathbf{8 6}$ ) in the context of periodic finite-gap potentials and by Pöschel and Trubowitz [106] and Ralston and Trubowitz [108] for various boundary value problems on compact intervals. As in the previous case, this method formally consists of two single commutations, but this time at different values of the spectral parameter $\mu$ and $\nu$. The resulting operator will have the Wronskian $W(\psi(\mu), \psi(\nu))$ 
in its denominator and it turns out that it will be well-defined as long as both $\mu$ and $\nu$ lie in the same spectral gap of $H$. This is related to the fact, that $(H-\mu)(H-\nu)$ will still be nonnegative under this assumption and hence can still be factorized as $(H-\mu)(H-\nu)=B^{*} B$, as explained later by Schmincke 119. Moreover, the fact that the zeros of the Wronskian of two solutions is related to the spectrum lead to the development of renormalized oscillation theory $[\mathbf{7 2}$ to be discussed in Section 6 below.

To explain the relevance of this method recall that an important role in inverse spectral theory is played by the so called Dirichlet data as introduced at the end of the previous section. The key result of $\mathbf{7 0}$. states that the above commutation method can be used to move a given Dirichlet eigenvalue to any other admissible position within its gap as well as change its sign. Hence it is also known as the Dirichlet deformation method. We refrain from further details here and refer to Fritz's own review [38] instead. For generalizations to other operators see [114, 115, 128, 129, 133 .

For further generalizations of commutation methods see $[\mathbf{8}, \mathbf{9}, \mathbf{3}$. Finally, these methods are also relevant in connection with inverse scattering theory (see, e.g., 1, 103 and the references cited therein) and yield a direct construction of $N$-soliton solutions relative to arbitrary background solutions of the (generalized) $\mathrm{KdV}$ hierarchy along the methods of $\mathbf{7 3}$ (see also $\mathbf{7 9}$ ). Generalizations to other integrable equations can be found in $[14,104,10,60,116,131$.

\section{Oscillation Theory}

Another area where Fritz had an important impact is oscillation theory. As indicated at the end of the previous section, a crucial ingredient in the development of the Dirichlet deformation method was the fact that the Wronskian of two solutions $W(\psi(\mu), \psi(\nu))$ is nonzero as long as there is no part of the spectrum inside the interval $(\mu, \nu)$. This observation naturally lead to the question how the number of points in the spectrum between $\mu$ and $\nu$ are related to the number of zeros of the Wronskian. This question was answered by Gesztesy, Simon, and Teschl in $\mathbf{7 2}$.

Denote by $W_{0}(f, g)$ be the number of zeros of the Wronskian in the open interval $(a, b)$ not counting multiplicities of zeros. Given $E_{1}<E_{2}$, we let $N_{0}\left(E_{1}, E_{2}\right)=$ dim ran $P_{\left(E_{1}, E_{2}\right)}(H)$ be the dimension of the spectral projection $P_{\left(E_{1}, E_{2}\right)}(H)$ of $H$. Then the main result from $[\mathbf{7 2}$ is the following:

Theorem $6.1(\mathbf{7 2}])$. Let $\psi_{-}(\lambda, x), \psi_{+}(\lambda, x)$ be solutions of $\tau \psi=\lambda \psi$ which are square integrable near $a, b$ and satisfy a possible boundary condition at $a, b, r e-$ spectively. (Such solutions will in general only exist inside a spectral gap). Suppose $E_{1}<E_{2}$. Then

$$
W_{0}\left(\psi_{-}\left(E_{1}\right), \psi_{+}\left(E_{2}\right)\right)=N_{0}\left(E_{1}, E_{2}\right) .
$$

This result constituted an important generalization suitable for counting the number of eigenvalues inside essential gaps. In fact, there has been considerable efforts to generalize classical oscillation theory to singular operators by Hartman and others around 1950. However, while these efforts were successful below the essential spectrum, they were only partly successful for counting eigenvalues in essential spectral gaps. For example Hartmann [84] could show that for a regular endpoint $a$ and a limit point endpoint $b$, counting the difference of zeros of the solutions $\psi_{-}\left(E_{2}, x\right)$ and $\psi_{-}\left(E_{1}, x\right)$ on $(a, c)$ will have $N_{0}\left(E_{1}, E_{2}\right)$ as its liminf when 
$c \rightarrow b$. As a simple example, the above theorem allows to extend this result to the case when $a$ is non-oscillatory (thus covering important examples like radial Schrödinger equations).

Finally let us outline a proof for the above result. The first step is to prove this result in the case where both endpoints are regular. This can be done using the usual Prüfer techniques and we refer to the textbook 135. for a simple proof. Next one can approximate $H$ on $(a, b)$ by regular operators on $(c, d) \subseteq(a, b)$. Again this technique is standard (for a nice review see [143) and the restricted operators will converge to $H$ in the strong resolvent sense. Since the spectrum cannot expand for such limits, one obtains $W_{0} \geq N_{0}$. To obtain the reverse inequality one uses a variational argument. To this end observe that at every zero of the Wronskian the two solutions $\psi_{-}\left(E_{1}\right)$ and $\psi_{+}\left(E_{2}\right)$ can be glued together (by scaling one to match the value of the other) to give a trial function in the domain of $H$ (since the derivatives will then automatically match at this point). Hence one obtains a $W_{0}$ dimensional space of trial functions $\psi$ satisfying

$$
\left\|\left(H-\frac{E_{2}-E_{1}}{2}\right) \psi\right\|<\frac{E_{2}-E_{1}}{2}\|\psi\|
$$

and the required reverse inequality $W_{0} \leq N_{0}$ follows from the spectral theorem. For further recent reviews of these methods we refer to the ones by Fritz [38] and Simon [125].

Again this result has triggered several extensions. In particular, it was shown by Krüger and Teschl [96] that one can take solutions of different operators if the right-hand side is interpreted as spectral shift between these two operators. We refer to [96, 97] and the references therein. The question whether eigenvalues accumulate at the boundary of an essential spectral gap based on these methods is considered in $\mathbf{9 8}, \mathbf{1 1 7}$. In this respect we should also mention the beautiful result by Fritz and Ünal $\mathbf{7 8}$ which gives the most general version of Kneser's theorem. Extensions to other operators can be found in [4, 29, 126, 127, 130.

\section{Non-self-adjoint operators}

While all results so far were concerned with self-adjoint operators, Fritz always was quite active in the area of non-self-adjoint operators as well. As a prototypical example we mention his beautiful work with Tkachenko [75, 76] solving the longstanding open question when a non-self-adjoint Hill operator is a spectral operator of scalar type in the sense of Dunford. This problem had been open for about 40 years.

We have already encountered Hill's equation (i.e, (3.1) with periodic potential $q(x+1)=q(x))$ in Section 4. But now we will allow $q$ to be complex valued (in addition it is assumed to be locally square integrable). Recall that one of the key objects from Floquet theory is the Floquet discriminant

$$
\Delta(z)=\frac{\theta(z, 1)+\phi^{\prime}(z, 1)}{2},
$$

where $\theta(z, x), \phi(z, x)$ is a fundamental system of solutions satisfying the initial conditions (3.2) at $a=0$. It was shown by Serov [122] that the spectrum of $H$ is given by $\sigma(H)=\{z \in \mathbb{C} \mid \Delta(z) \in[-1,1]\}$. In particular, the spectrum consists of a number of analytic arcs which, however, might intersect in inner points $\mathbf{1 0 5}$. 
The following version of this criterion involves the spectrum $\sigma(H)$ of $H$, the Dirichlet spectrum $\left\{\mu_{k}\right\}_{k \in \mathbb{N}}$, the periodic spectrum $\left\{E_{k}(0)\right\}_{k \in \mathbb{N}_{0}}$, and the antiperiodic spectrum $\left\{E_{k}(\pi)\right\}_{k \in \mathbb{N}_{0}}$, and is connected with the algebraic and geometric multiplicities of the eigenvalues in the sets $\sigma(H(t))$, where $H(t), t \in[0,2 \pi]$, denotes the densely defined closed realization of (3.1) in $L^{2}(0,1)$ in terms of the $t$-dependent boundary conditions $f(1)=\mathrm{e}^{\mathrm{i} t} f(0), f^{\prime}(1)=\mathrm{e}^{\mathrm{i} t} f^{\prime}(0), t \in[0,2 \pi]$. It is known that the union of the periodic and antiperiodic spectra is formed by the numbers

$$
\begin{aligned}
& \lambda_{k}^{ \pm}=\left(k+\frac{\int_{0}^{1} q(x) d x}{2 k}+\frac{s_{k}^{ \pm}}{k}\right)^{2}, k \in \mathbb{N}, \quad \sum_{k \in \mathbb{N}}\left|s_{k}^{ \pm}\right|^{2}<\infty, \\
& \left\{E_{k}(0)\right\}_{k \in \mathbb{N}_{0}}=\left\{\lambda_{0}^{+}, \lambda_{2 k}^{+}, \lambda_{2 k}^{-}\right\}_{k \in \mathbb{N}}, \quad\left\{E_{k}(\pi)\right\}_{k \in \mathbb{N}_{0}}=\left\{\lambda_{2 k+1}^{+}, \lambda_{2 k+1}^{-}\right\}_{k \in \mathbb{N}_{0}},
\end{aligned}
$$

THEOREM 7.1. A Hill operator $H$ is a spectral operator of scalar type if and only if the following conditions $(i)$ and $(i i)$ are satisfied:

(i) For all $t \in[0,2 \pi]$ and all $E_{k}(t) \in \sigma(H(t))$, each root function of the operator $H(t)$ associated with $E_{k}(t)$ is an eigenfunction of $H(t)$.

(ii) Let

$$
\mathcal{Q}=\left\{k \in \mathbb{N} \mid d_{k}=\operatorname{dist}\left(\delta_{k}, \sigma(H)\right)>0\right\}
$$

Then

$$
\sup _{k \in \mathcal{Q}} \frac{\left|\lambda_{k}^{+}-\lambda_{k}^{-}\right|}{\operatorname{dist}\left(\delta_{k}, \sigma(H)\right)}<\infty, \quad \sup _{k \in \mathcal{Q}} \frac{\left|\mu_{k}-\lambda_{k}^{-}\right|}{\operatorname{dist}\left(\delta_{k}, \sigma(H)\right)}<\infty, \quad \sup _{k \in \mathcal{Q}} \frac{\left|\mu_{k}-\lambda_{k}^{+}\right|}{\operatorname{dist}\left(\delta_{k}, \sigma(H)\right)}<\infty .
$$

Here a root function of $H(t)$ associated with the eigenvalue $E_{k}(t)$ denotes any element $\psi$ satisfying $\left(H(t)-E_{k}(t)\right)^{m} \psi=0$ for some $m \in \mathbb{N}$ (i.e., any element in the algebraic eigenspace of $H(t)$ corresponding to $\left.E_{k}(t)\right)$. Of course, the conditions $(i)$ and $(i i)$ are always satisfied in the self-adjoint context where $q$ is real-valued.

It can be shown that the conditions $(i)$ and $(i i)$ imposed in Theorem 7.1 yield the remarkable fact that the property of a Hill operator being a spectral operator is independent of smoothness (or even analyticity) properties of the potential $q$. In addition, also a functional model for periodic Schrödinger operators that are spectral operators of scalar type was established and the corresponding eigenfunction expansion was developed in 75, 76.

In their recent paper [77, under the assumption that $q \in L^{2}(0,1)$, necessary and sufficient conditions in terms of spectral data for (non-self-adjoint) Schrödinger operators in $L^{2}(0,1)$ with periodic and antiperiodic boundary conditions to possess a Riesz basis of root vectors were derived. Without entering details, we mention that this problem generated an enormous amount of interest and remained open for a long time. Their key result reads:

TheOREm 7.2. Assume $q \in L^{2}(0,1)$, then the following results hold: (i) The system of root vectors of $H(0)$ contains a Riesz basis in $L^{2}(0,1)$ if and only if

$$
\sup _{\substack{k \in \mathbb{N} \\ \lambda_{2 k}^{+} \neq \lambda_{2 k}^{-}}} \frac{\left|\mu_{2 k}-\lambda_{2 k}^{ \pm}\right|}{\left|\lambda_{2 k}^{+}-\lambda_{2 k}^{-}\right|}<\infty
$$


(ii) The system of root vectors of $H(\pi)$ contains a Riesz basis in $L^{2}(0,1)$ if and only if

$$
\sup _{\substack{k \in \mathbb{N}, \lambda_{2 k+1}^{+} \neq \lambda_{2 k+1}^{-}}} \frac{\left|\mu_{2 k+1}-\lambda_{2 k+1}^{ \pm}\right|}{\left|\lambda_{2 k+1}^{+}-\lambda_{2 k+1}^{-}\right|}<\infty .
$$

Here $\sup _{k \in \mathbb{N}, \lambda_{j}^{+} \neq \lambda_{j}^{-}}$signifies that all subscripts $j \in \mathbb{N}$ in (7.6) and (7.7) for which $\lambda_{j}^{+}$and $\lambda_{j}^{-}$coincide are simply excluded from the supremum considered.

One observes that only the simple periodic (resp., antiperiodic) eigenvalues enter in the necessary and sufficient conditions (7.6) (resp., (7.7)) for the existence of a Riesz basis of root vectors of $H(0)$ (resp., $H(\pi)$ ). The multiple periodic (resp., antiperiodic) eigenvalues play no role in deciding whether or not the system of root vectors of $H(0)$ (resp., $H(\pi)$ ) constitutes a Riesz basis in $L^{2}(0,1)$. In addition, only every other Dirichlet eigenvalue (i.e., half the Dirichlet spectrum) enters the criterion (7.6) (resp., (7.7)).

For additional detailed results in this direction we also refer to [23] and the extensive literature cited therein.

Acknowledgments. We thank Barry Simon for valuable comments improving the presentation of the material.

\section{References}

[1] T. Aktosun, M. Klaus, and C. van der Mee, Scattering and inverse scattering in onedimensional nonhomogeneous media, J. Math. Phys. 32, 1717-1744 (1992).

[2] S. Albeverio, F. Gesztesy, R. Høegh-Krohn, and H. Holden, Solvable Models in Quantum Mechanics, 2nd ed., AMS Chelsea Publishing, Providence, RI, 2005.

[3] S. Albeverio, R. Hryniv, and Y. Mykytyuk, Factorisation of non-negative Fredholm operators and inverse spectral problems for Bessel operators, Integral Equations Operator Theory 64, 301-323 (2009).

[4] K. Ammann and G. Teschl, Relative oscillation theory for Jacobi matrices, in Proceedings of the 14th International Conference on Difference Equations and Applications, M. Bohner (ed.) et al., 105-115, Uğur-Bahçeşehir University Publishing Company, Istanbul, 2009.

[5] M. S. Ashbaugh, F. Gesztesy, M. Mitrea, and G. Teschl, Spectral theory for perturbed Krein Laplacians in nonsmooth domains, Adv. Math. 223, 1372-1467 (2010).

[6] B. Baumgartner, Level comparison theorems, Ann. Phys. (N.Y.) 168, 484-526 (1986).

[7] P. Bechouche, N. J. Mauser, and F. Poupaud, (Semi)-nonrelativistic limits of the Dirac equation with external time-dependent electromagnetic field, Comm. Math. Phys. 197, 405-425 (1998).

[8] P. A. Binding, P. J. Browne, and B. A. Watson, Darboux transformations and the factorization of generalized Sturm-Liouville problems, Proc. Roy. Soc. Edinburgh Sect. A 140, 1-29 (2010).

[9] A. Boumenir and V. K. Tuan, A trace formula and Schmincke inequality on the half-line, Proc. Amer. Math. Soc. 137, 1039-1049 (2009).

[10] W. Bulla, F. Gesztesy, H. Holden, and G. Teschl, Algebro-geometric quasi-periodic finitegap solutions of the Toda and Kac-van Moerbeke hierarchies, Memoirs of the Amer. Math. Soc. 135, no. 641, 1998.

[11] W. Bulla, F. Gesztesy, and K. Unterkofler, On relativistic energy band corrections in the presence of periodic potentials, Lett. Math. Phys. 15, 313-324 (1988).

[12] W. Bulla, F. Gesztesy, and K. Unterkofler, Holomorphy of the scattering matrix with respect to $c^{-2}$ for Dirac operators and an explicit treatment of relativistic corrections, Comm. Math. Phys. 144, 391-416 (1992).

[13] M. Buys and A. Finkel, The inverse periodic problem for Hill's equation with a finite-gap potential, J. Diff. Eqs. 55, 257-275 (1984). 
[14] R. C. Cascaval, F. Gesztesy, H. Holden, and Y. Latushkin, Spectral analysis of Darboux transformations for the focusing NLS hierarchy, J. Anal. Math. 93, 139-197 (2004).

[15] R. J. Cirincione and P. R. Chernoff, Dirac and Klein-Gordon equations: Convergence of solutions in the nonrelativistic limit, Comm. Math. Phys. 79, 33-46 (1981).

[16] S. Clark and F. Gesztesy, Weyl-Titchmarsh M-function asymptotics, local uniqueness results, trace formulas, and Borg-type theorems for Dirac operators, Trans. Amer. Math. Soc. 354, 3475-3534 (2002).

[17] S. Clark, F. Gesztesy, and W. Renger, Trace formulas and Borg-type theorems for matrixvalued Jacobi and Dirac finite difference operators, J. Differential Equations 219, no. 1, 144-182 (2005).

[18] M. M. Crum, Associated Sturm-Liouville systems, Quart. J. Math. Oxford (2) 6, 121$127(1955)$

[19] G. Darboux, Sur une proposition relative aux équations linéaires, C. R. Acad. Sci. (Paris) 94, 1456-1459 (1882).

[20] P. A. Deift, Applications of a commutation formula, Duke Math. J. 45, 267-310 (1978).

[21] P. Deift and E. Trubowitz, Inverse scattering on the line, Comm. Pure Appl. Math. 32, 121-251 (1979).

[22] R. del Rio, F. Gesztesy, and B. Simon, Inverse spectral analysis with partial information on the potential, III. Updating boundary conditions, Intl. Math. Research Notices 1997, 751-758 (1997).

[23] P. Djakov and B. Mityagin, Criteria for existence of Riesz bases consisting of root functions of Hill and 1d Dirac operators, J. Funct. Anal. 263, 2300-2332 (2012).

[24] J. Eckhardt, Inverse uniqueness results for Schrödinger operators using de Branges theory, Complex Anal. Oper. Theory (to appear).

[25] J. Eckhardt, F. Gesztesy, R. Nichols, and G. Teschl, Weyl-Titchmarsh theory for SturmLiouville operators with distributional potentials, Opuscula Math. 33, 467-563 (2013).

[26] J. Eckhardt, F. Gesztesy, R. Nichols, and G. Teschl, Inverse spectral theory for SturmLiouville operators with distributional potentials, arXiv:1210.7628

[27] J. Eckhardt and G. Teschl, Uniqueness results for one-dimensional Schrödinger operators with purely discrete spectra, Trans. Amer. Math. Soc. 365, 3923-3942 (2013).

[28] J. Eckhardt and G. Teschl, Sturm-Liouville operators with measure-valued coefficients, J. Analyse Math. (to appear).

[29] J. Elyseeva, On relative oscillation theory for symplectic eigenvalue problems, Appl. Math. Lett. 23, 1231-1237 (2010).

[30] A. Finkel, E. Isaacson, and E. Trubowitz, An explicit solution of the inverse problem for Hill's equation, SIAM J. Math. Anal. 18, 46-53 (1987).

[31] C. Fulton, Titchmarsh-Weyl m-functions for second order Sturm-Liouville problems, Math. Nachr. 281, 1417-1475 (2008).

[32] C. Fulton and H. Langer, Sturm-Liouville operators with singularities and generalized Nevanlinna functions, Complex Anal. Oper. Theory 4, 179-243 (2010).

[33] C. Fulton, H. Langer, and A. Luger, Mark Krein's method of directing functionals and singular potentials, Math. Nachr. 285, 1791-1798 (2012).

[34] C. S. Gardner, J. M. Greene, M. D. Kruskal, and R. M. Miura, Korteweg-de Vries equation and generalizations. VI. Methods for exact solution, Comm. Pure Appl. Math. 27, 97-133 (1974).

[35] I. M. Gel'fand and B. M. Levitan, On the determination of a differential equation from its spectral function, Amer. Math. Soc. Transl. Ser 2, 1, 253-304 (1955).

[36] F. Gesztesy, A complete spectral characterization of the double commutation method, J. Funct. Anal. 117, 401-446 (1993).

[37] F. Gesztesy, New trace formulas for Schrödinger operators, Evolution equations (Baton Rouge, LA, 1992), 201-221, Lecture Notes in Pure and Appl. Math., 168, Dekker, New York, 1995.

[38] F. Gesztesy, Inverse spectral theory as influenced by Barry Simon in "Spectral theory and mathematical physics: a Festschrift in honor of Barry Simon's 60th birthday", 741820, Proc. Sympos. Pure Math., 76, Part 2, Amer. Math. Soc., Providence, RI, 2007.

[39] F. Gesztesy, H. Grosse, and B. Thaller, Spectral concentration in the nonrelativistic limit, Phys. Lett. B 116, 155-157 (1982). 
[40] F. Gesztesy, H. Grosse, and B. Thaller, Efficient method for calculating relativistic corrections for spin-1/2 particles, Phys. Rev. Lett. 50, 625-628 (1983).

[41] F. Gesztesy, H. Grosse, and B. Thaller, A rigorous approach to relativistic corrections of bound state energies for spin-1/2 particles, Ann. Inst. Henri Poincare A 40, 159-174 (1984).

[42] F. Gesztesy, H. Grosse, and B. Thaller, On relativistic corrections to bound state energies for two-fermion systems, Phys. Rev. D 30, 2189-2193 (1984).

[43] F. Gesztesy, H. Grosse, and B. Thaller, First-order relativistic corrections and spectral concentration, Adv. Appl. Math. 6, 159-176 (1985).

[44] F. Gesztesy and H. Holden, On trace formulas for Schrödinger-type operators, in Multiparticle quantum scattering with applications to nuclear, atomic and molecular physics, 121-145, IMA Vol. Math. Appl. 89, Springer, New York, 1997.

[45] F. Gesztesy and H. Holden, On new trace formulae for Schrödinger operators, Acta Appl. Math. 39, 315-333 (1995).

[46] F. Gesztesy and H. Holden, Trace formulas and conservation laws for nonlinear evolution equations, Rev. Math. Phys. 6, 51-95 (1994).

[47] F. Gesztesy and H. Holden, Errata: "Trace formulas and conservation laws for nonlinear evolution equations", Rev. Math. Phys. 6, 673 (1994).

[48] F. Gesztesy and H. Holden, Soliton Equations and Their Algebro-Geometric Solutions. Volume I: $(1+1)$-Dimensional Continuous Models. Cambridge Studies in Advanced Mathematics 79, Cambridge University Press, Cambridge, 2003.

[49] F. Gesztesy, H. Holden, J. Michor, and G. Teschl, Soliton Equations and Their AlgebroGeometric Solutions. Volume II: (1+1)-Dimensional Discrete Models, Cambridge Studies in Advanced Mathematics 114, Cambridge University Press, Cambridge, 2008.

[50] F. Gesztesy, H. Holden, and B. Simon, Absolute summability of the trace relation for certain Schrödinger operators, Comm. Math. Phys. 168, 137-161 (1995).

[51] F. Gesztesy, H. Holden, B. Simon, and Z. Zhao, Trace formulae and inverse spectral theory for Schrödinger operators, Bull. Amer. Math. Soc. (N.S.) 29, 250-255 (1993).

[52] F. Gesztesy, H. Holden, B. Simon, and Z. Zhao, Higher order trace relations for Schrödinger operators, Rev. Math. Phys. 7 (1995), no. 6, 893-922.

[53] F. Gesztesy, H. Holden, B. Simon, and Z. Zhao, A trace formula for multidimensional Schrödinger operators, J. Funct. Anal. 141, 449-465 (1996).

[54] F. Gesztesy, N. J. Kalton, K. A. Makarov, and E. Tsekanovskii, Some Applications of Operator-Valued Herglotz Functions, in Operator Theory, System Theory and Related Topics. The Moshe Livšic Anniversary Volume, D. Alpay and V. Vinnikov (eds.), 271321, Oper. Theory Adv. Appl., 123, Birkhäuser, Basel, 2001.

[55] F. Gesztesy, K. Maddaly, and G. Teschl, On isospectral sets of Jacobi operators, Comm. Math. Phys. 181, 631-645 (1996).

[56] F. Gesztesy and M. Mitrea, Generalized Robin boundary conditions, Robin-to-Dirichlet maps, and Krein-type resolvent formulas for Schrödinger operators on bounded Lipschitz domains, in Perspectives in Partial Differential Equations, Harmonic Analysis and Applications: A Volume in Honor of Vladimir G. Maz'ya's 70th Birthday, D. Mitrea and M. Mitrea (eds.), 105-173, Proc. Sympos. Pure Math., 79, Amer. Math. Soc., Providence, RI, 2008.

[57] F. Gesztesy and M. Mitrea, A description of all self-adjoint extensions of the Laplacian and Krein-type resolvent formulas on non-smooth domains, J. Analyse Math. 113, 53$172(2011)$

[58] F. Gesztesy, M. Mitrea, and M. Zinchenko, Variations on a Theme of Jost and Pais, J. Funct. Anal. 253, 399-448 (2007).

[59] F. Gesztesy, R. Ratnaseelan, and G. Teschl, The KdV hierarchy and associated trace formulas, in Recent developments in operator theory and its applications, 125-163, Oper. Theory Adv. Appl., 87, Birkhäuser, Basel, 1996.

[60] F. Gesztesy and W. Renger, New classes of Toda soliton solutions, Comm. Math. Phys. 184, 27-50 (1997).

[61] F. Gesztesy, W. Schweiger, and B. Simon, Commutation methods applied to the mKdVequation, Trans. Amer. Math. Soc. 324, 465-525 (1991).

[62] F. Gesztesy and B. Simon, The xi-function, Acta Math. 176, 49-71 (1996). 
[63] F. Gesztesy and B. Simon, Inverse spectral analysis with partial information on the potential, I. The case of an a.c. component in the spectrum, Helv. Phys. Acta 70,66-71 (1997).

[64] F. Gesztesy and B. Simon, On the determination of a potential from three spectra, Amer. Math. Soc. Transl. (2) 189, 85-92 (1999).

[65] F. Gesztesy and B. Simon, Inverse spectral analysis with partial information on the potential, II. The case of discrete spectrum, Trans. Amer. Math. Soc. 352, 2765-2787 (2000).

[66] F. Gesztesy and B. Simon, On local Borg-Marchenko uniqueness results, Comm. Math. Phys. 211, 273-287 (2000).

[67] F. Gesztesy and B. Simon, Uniqueness theorems in inverse spectral theory for onedimensional Schrödinger operators, Trans. Amer. Math. Soc. 348, 349-373 (1996).

[68] F. Gesztesy and B. Simon, A new approach to inverse spectral theory, II. General real potentials and the connection to the spectral measure, Ann. of Math. 152, 593-643 (2000).

[69] F. Gesztesy, B. Simon, and A. Pushnitski, On the Koplienko spectral shift function, I. Basics, Zh. Mat. Fiz. Anal. Geom. 4, 63-107 (2008).

[70] F. Gesztesy, B. Simon, and G. Teschl, Spectral deformations of one-dimensional Schrödinger operators, J. d'Analyse Math. 70, 267-324 (1996).

[71] F. Gesztesy and G. Teschl, On the double commutation method, Proc. Amer. Math. Soc. 124, 1831-1840 (1996).

[72] F. Gesztesy, B. Simon, and G. Teschl, Zeros of the Wronskian and renormalized oscillation theory, Am. J. Math. 118, 571-594 (1996).

[73] F. Gesztesy and R. Svirsky, ( $m$ )KdV-Solitons on the background of quasi-periodic finitegap solutions, Memoirs Amer. Math. Soc. 118, no. 563 (1995).

[74] F. Gesztesy and G. Teschl, Commutation methods for Jacobi operators, J. Diff. Eqs. 128, 252-299 (1996).

[75] F. Gesztesy and V. Tkachenko, When is a non-self-adjoint Hill operator a spectral operator of scalar type?, C. R. Math. Acad. Sci. Paris 343, 239-242 (2006).

[76] F. Gesztesy and V. Tkachenko, A criterion for Hill operators to be spectral operators of scalar type, J. Anal. Math. 107, 287-353 (2009).

[77] F. Gesztesy and V. Tkachenko, A Schauder and Riesz basis criterion for non-self-adjoint Schrödinger operators with periodic and antiperiodic boundary conditions, J. Diff. Eqs. 253, 400-437 (2012).

[78] F. Gesztesy and M. Ünal, Perturbative oscillation criteria and Hardy-type inequalities, Math. Nachr. 189, 121-144 (1998).

[79] F. Gesztesy and K. Unterkofler, Isospectral deformations for Sturm-Liouville and Diractype operators and associated nonlinear evolution equations, Rep. Math. Phys. 31, 113137 (1992).

[80] F. Gesztesy and R. Weikard, Spectral deformations and soliton equations, in "Differential Equations with Applications in Mathematical Physics", W. F. Ames, E. M. Harrell, J. V. Herod (eds.), 101-139, Academic Press, Boston, 1993.

[81] F. Gesztesy and Z. Zhao, On critical and subcritical Sturm-Liouville operators, J. Funct. Anal. 98, 311-345 (1991).

[82] F. Gesztesy and M. Zinchenko, On spectral theory for Schrödinger operators with strongly singular potentials, Math. Nachr. 279, 1041-1082 (2006).

[83] D. R. Grigore, G. Nenciu, and R. Purice, On the nonrelativistic limit of the Dirac Hamiltonian, Ann. Inst. Henri Poincare A 51, 231-263 (1989).

[84] P. Hartman, A characterization of the spectra of one-dimensional wave equations, Am. J. Math. 71, 915-920 (1949).

[85] W. Hunziker, On the nonrelativistic limit of the Dirac theory, Comm. Math. Phys. 40, 215-222 (1975).

[86] K. Iwasaki, Inverse problem for Sturm-Liouville and Hill's equations, Ann. Mat. Pura Appl. 149, 185-206 (1987).

[87] C. G. J. Jacobi, Zur Theorie der Variationsrechnung und der Differentialgleichungen, J. Reine Angew. Math. 17, 68-82 (1837). 
[88] I. S. Kac, The existence of spectral functions of generalized second order differential systems with boundary conditions at the singular end, AMS Translations (2) 62, 204262 (1967).

[89] I. Kay and H. E. Moses, Reflectionless transmission through dielectrics and scattering potentials, J. Appl. Phys. 27, 1503-1508 (1956).

[90] T. Kato, Perturbation theory for linear operators, 2nd ed., Springer-Verlag, New York, Berlin, 1980.

[91] K. Kodaira, The eigenvalue problem for ordinary differential equations of the second order and Heisenberg's theory of S-matrices, Amer. J. Math. 71, 921-945 (1949).

[92] A. Kostenko, A. Sakhnovich, and G. Teschl, Weyl-Titchmarsh theory for Schrödinger operators with strongly singular potentials, Int. Math. Res. Notices 2012, no. 8, 16991747.

[93] A. Kostenko, A. Sakhnovich, and G. Teschl, Commutation methods for Schrödinger operators with strongly singular potentials, Math. Nachr. 285, 392-410 (2012).

[94] A. Kostenko and G. Teschl, On the singular Weyl-Titchmarsh function of perturbed spherical Schrödinger operators, J. Diff. Eqs. 250, 3701-3739 (2011).

[95] A. Kostenko and G. Teschl, Spectral asymptotics for perturbed spherical Schrödinger operators, Comm. Math. Phys. (to appear). DOI 10.1007/s00220-013-1698-x

[96] H. Krüger and G. Teschl, Relative oscillation theory, zeros of the Wronskian, and the spectral shift function, Comm. Math. Phys. 287, 613-640 (2009).

[97] H. Krüger and G. Teschl, Relative oscillation theory for Sturm-Liouville operators extended, J. Funct. Anal. 254, 1702-1720 (2008).

[98] H. Krüger and G. Teschl, Effective Prüfer angles and relative oscillation criteria, J. Diff. Eq. 245, 3823-3848 (2008).

[99] P. Kurasov and A. Luger, An operator theoretic interpretation of the generalized Titchmarsh-Weyl coefficient for a singular Sturm-Liouville problem, Math. Phys. Anal. Geom. 14, 115-151 (2011).

[100] E. A. Kuznetsov and A. V. Mikhailov, Stability of solitary waves in nonlinear weakly dispersive media, Sov. Phys. JETP 40, 855-859 (1975).

[101] B. M. Levitan, Inverse Sturm-Liouville Problems, VNU Science Press, Utrecht, 1987.

[102] B. M. Levitan and I. S. Sargsjan, Sturm-Liouville and Dirac Operators, Kluwer Academic Publishers, Dordrecht, 1991.

[103] V. A. Marchenko, Sturm-Liouville Operators and Applications, Birkhäuser, Basel, 1986.

[104] M. Ohmiya and M. Urakubo, Isospectral prosperty of double Darboux transformation, Osaka J. Math. 43, 877-893 (2006).

[105] L. A. Pastur and V. A. Tkachenko, Geometry of the spectrum of the one-dimensional Schrödinger equation with a periodic complex-valued potential, Math. Notes 50, 10451050 (1991).

[106] J. Pöschel and E. Trubowitz, Inverse Spectral Theory, Academic Press, Boston, 1987.

[107] A. Pushnitski and I. Sorrell, High energy asymptotics and trace formulas for the perturbed harmonic oscillator, Ann. H. Poincare 7, 1-17 (2005).

[108] J. Ralston and E. Trubowitz, Isospectral sets for boundary value problems on the unit interval, Ergod. Th. Dynam. Sys. 8, 301-358 (1988).

[109] M. Reed and B. Simon, Methods of modern mathematical physics. Vol. IV: Analysis of operators, Academic Press, New York, 1978.

[110] A. V. Rybin and M. A. Sall', Solitons of the Korteweg-de Vries equation on the background of a known solution, Theoret. Math. Phys. 63, 545-550 (1985).

[111] A. Rybkin, On the trace approach to the inverse scattering problem in dimension, SIAM J. Math. Anal. 32, 1248-1264 (2001).

[112] A. Rybkin, Some new and old asymptotic representations of the Jost solution and the Weyl m-function for Schrödinger operators on the line, Bull. London Math. Soc. 34, 61-72 (2002).

[113] A. Rybkin, Necessary and sufficient conditions for absolute summability of the trace formulas for certain one dimensional Schrödinger operators, Proc. Amer. Math. Soc. 131, 219-229 (2002).

[114] A. Sakhnovich, Iterated Backlund-Darboux transform for canonical systems, J. Funct. Anal. 144, 359-370 (1997). 
[115] A. L. Sakhnovich, Generalized Bäcklund-Darboux transformation: spectral properties and nonlinear equations, J. Math. Anal. Appl. 262, 274-306 (2001).

[116] A. L. Sakhnovich, Discrete canonical system and non-abelian Toda lattice: BäcklundDarboux transformation, Weyl functions, and explicit solutions, Math. Nachr. 280, 631653 (2007).

[117] K. M. Schmidt, An application of the Gesztesy-Simon-Teschl oscillation theory to a problem in differential geometry, J. Math. Anal. Appl. 261, 61-71 (2001).

[118] U.-W. Schmincke, On Schrödinger's factorization method for Sturm-Liouville operators, Proc. Roy. Soc. Edinburgh 80A, 67-84 (1978).

[119] U.-W. Schmincke, On a paper by Gesztesy, Simon, and Teschl concerning isospectral deformations of ordinary Schrödinger operators, J. Math. Anal. Appl. 277, 51-78 (2003).

[120] A.Y. Schoene, On the nonrelativistic limits of the Klein-Gordon and Dirac equations, J. Math. Anal. Appl. 71, 36-47 (1979).

[121] P. Schwerdtfeger (ed.), Relativistic electronic structure theory - part 1. Fundamentals, Elsevier Science, 2002.

[122] M.I. Serov, Certain properties of the spectrum of a non-selfadjoint differential operator of the second order, Sov. Math. Dokl. 1, 190-192 (1960).

[123] G.L. Sewell, An appropriate relation between the energy levels of a particle in a field of given potential energy, calculated in the relativistic and non-relativistic theories, Proc. Cambridge Phil. Soc. 45, 631-637 (1949).

[124] T. Shirai, A trace formula for discrete Schrödinger operators, Publ. Res. Inst. Math. Sci. 34, 27-41 (1998).

[125] B. Simon, Sturm oscillation and comparison theorems, in "Sturm-Liouville Theory: Past and Present", 29-43, Birkhäuser Verlag, Basel, 2005.

[126] R. Stadler and G. Teschl, Relative oscillation theory for Dirac operators, J. Math. Anal. Appl. 371, 638-648 (2010).

[127] G. Teschl, Oscillation theory and renormalized oscillation theory for Jacobi operators, J. Diff. Eq. 129, 532-558 (1996).

[128] G. Teschl, Spectral deformations of Jacobi operators, J. Reine und Angew. Math. 491, 1-15 (1997).

[129] G. Teschl, Deforming the point spectra of one-dimensional Dirac operators, Proc. Amer. Math. Soc. 126, 2873-2881 (1998).

[130] G. Teschl, Renormalized oscillation theory for Dirac operators, Proc. Amer. Math. Soc. 126, 1685-1695 (1998).

[131] G. Teschl, Trace formulas and inverse spectral theory for Jacobi operators, Comm. Math. Phys. 196, 175-202 (1998).

[132] G. Teschl, On the Toda and Kac-van Moerbeke hierarchies, Math. Z. 231, 325-344 (1999).

[133] G. Teschl, Jacobi Operators and Completely Integrable Nonlinear Lattices, Math. Surv. and Mon. 72, Amer. Math. Soc., Rhode Island, 2000.

[134] G. Teschl, Mathematical Methods in Quantum Mechanics; With Applications to Schrödinger Operators, Graduate Studies in Mathematics 99, Amer. Math. Soc., Rhode Island, 2009.

[135] G. Teschl, Ordinary Differential Equations and Dynamical Systems, Graduate Studies in Mathematics 140, Amer. Math. Soc., Rhode Island, 2012.

[136] B. Thaller, The Dirac equation, Texts and Monographs in Physics, Springer, Berlin, Heidelberg, New York, 1992.

[137] E.C. Titchmarsh, On the relation between the eigenvalues in relativistic and nonrelativistic quantum mechanics, Proc. Roy. Soc. A 266, 33-46 (1962).

[138] K. Veselic, The nonrelativistic limit of the Dirac equation and the spectral concentration, Glasnik Matematički Ser. III 4, 231-241 (1969).

[139] K. Veselić, Perturbation of pseudoresolvents and analyticity in $1 / c$ of relativistic quantum mechanics, Comm. Math. Phys. 22, 27-43 (1971).

[140] J. Weidmann, Linear Operators in Hilbert Spaces, Springer, New York, 1980.

[141] J. Weidmann, Spectral Theory of Ordinary Differential Operators, Lecture Notes in Mathematics 1258, Springer, Berlin, 1987.

[142] J. Weidmann, Lineare Operatoren in Hilberträumen, Teil 2: Anwendungen, B. G. Teubner, Stuttgart, 2003. 
[143] J. Weidmann, Spectral theory of Sturm-Liouville operators; approximation by regular problems, in "Sturm-Liouville Theory: Past and Present", 75-98, Birkhäuser Verlag, Basel, 2005.

[144] A. Wiegner, Über den nichtrelativistischen Grenzwert der Eigenwerte der Diracgleichung, Master's thesis, Fernuniversität-Gesamthochschule Hagen, Hagen, Germany, 1984.

[145] M. Zinchenko, Trace formulas and a Borg-type theorem for CMV operators with matrixvalued coefficients, Math. Nachr. 283, 312-329 (2010).

Faculty of Mathematics, University of Vienna, Nordbergstrasse 15, 1090 Wien, Austria, and International Erwin Schrödinger Institute for Mathematical Physics, Boltzmanngasse 9, 1090 Wien, Austria

E-mail address: Gerald.Teschl@univie.ac.at

$U R L:$ http://www.mat.univie.ac.at/ gerald/

Vorarlberg University of Applied Sciences, Hochschulstr. 1, 6850 Dornbirn, AusTRIA

E-mail address: Karl.Unterkofler@fhv.at

$U R L:$ https://homepages.fhv.at/ku/ 